\title{
Making Maps by Hand
}

A FEW YEARS AGO, I began creating some small maps on paper, each one $5 \times 5$ ", drawn in faint graphite and delicate watercolour. This project was casual, with the maps made quickly and even carelessly. The results were unpolished and sometimes even illegible, but they maintain a freshness that every map I've made since has been envious of. Half of the maps were of highway interchanges, the other half of rivers. I was very taken with the similarity in shape between large highways and large rivers. Artificial and natural travelways, both intent on uninterrupted flow.

Drawing these maps led to many thoughts, observations, questions, and comparisons. Compare, for example, the wide loose braid of the Lena as it spreads over the taiga lowlands, to the artificial spreading of the Nile through a dense network of irrigation channels. Or an open crosshatch of highways in Ontario to an uncomfortably tight knot of converging roads in the mountains of Japan.

There are cultural and historical differences to be observed as well as physical restrictions. Four-leaf clovers abound in North America, while loops and whirlpools are common in China. I am confident that the interchanges of Dubai were designed to appear elegant from the sky.

Cities like New York, which were well established before the rise of car culture, crowded in their on-ramps and off-ramps wherever they could fit them, while cities like Houston, which grew up alongside the automobile, walled themselves in behind wide moats of road.

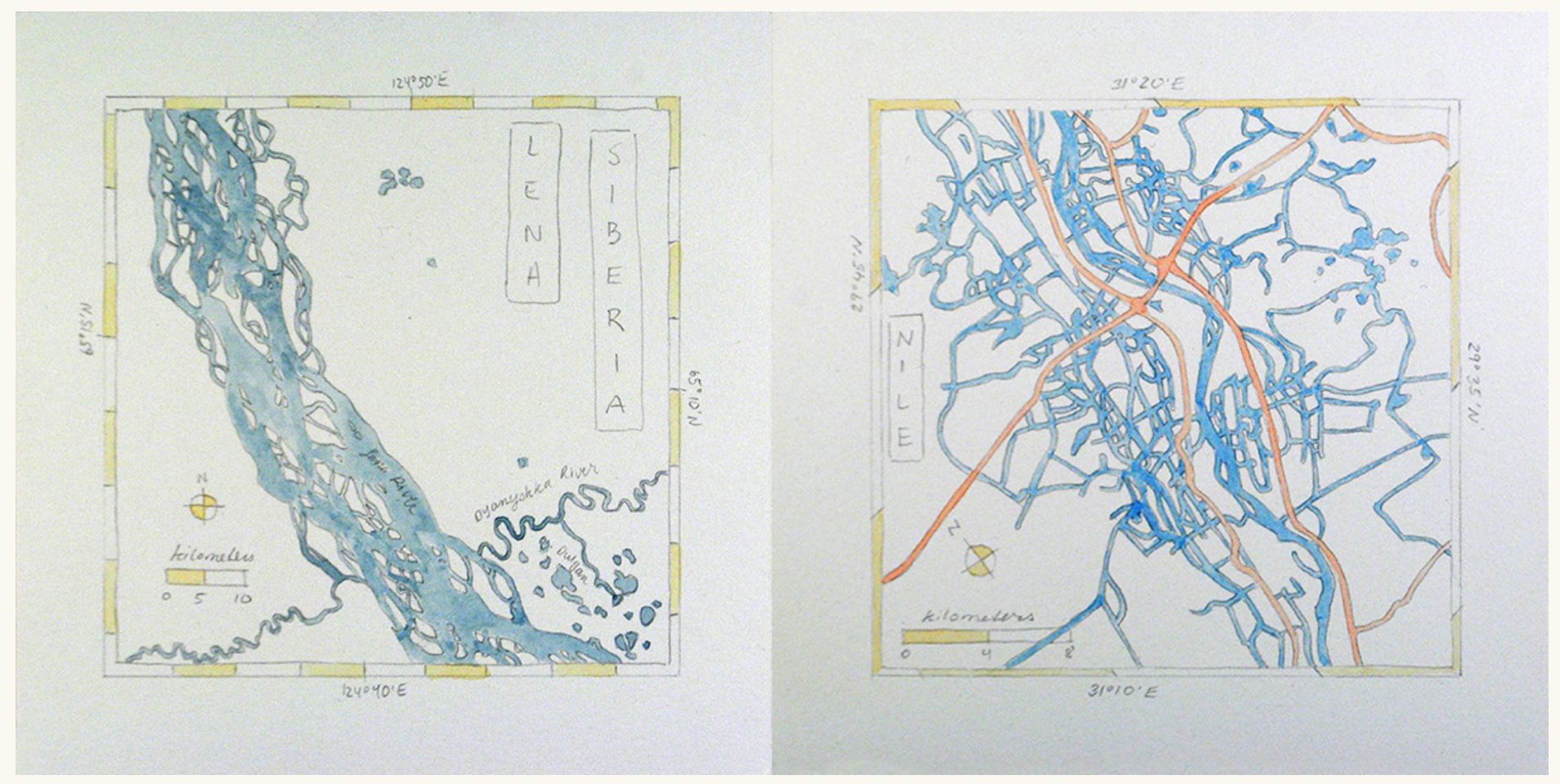

The braid of the Lena and the channels of the Nile. 

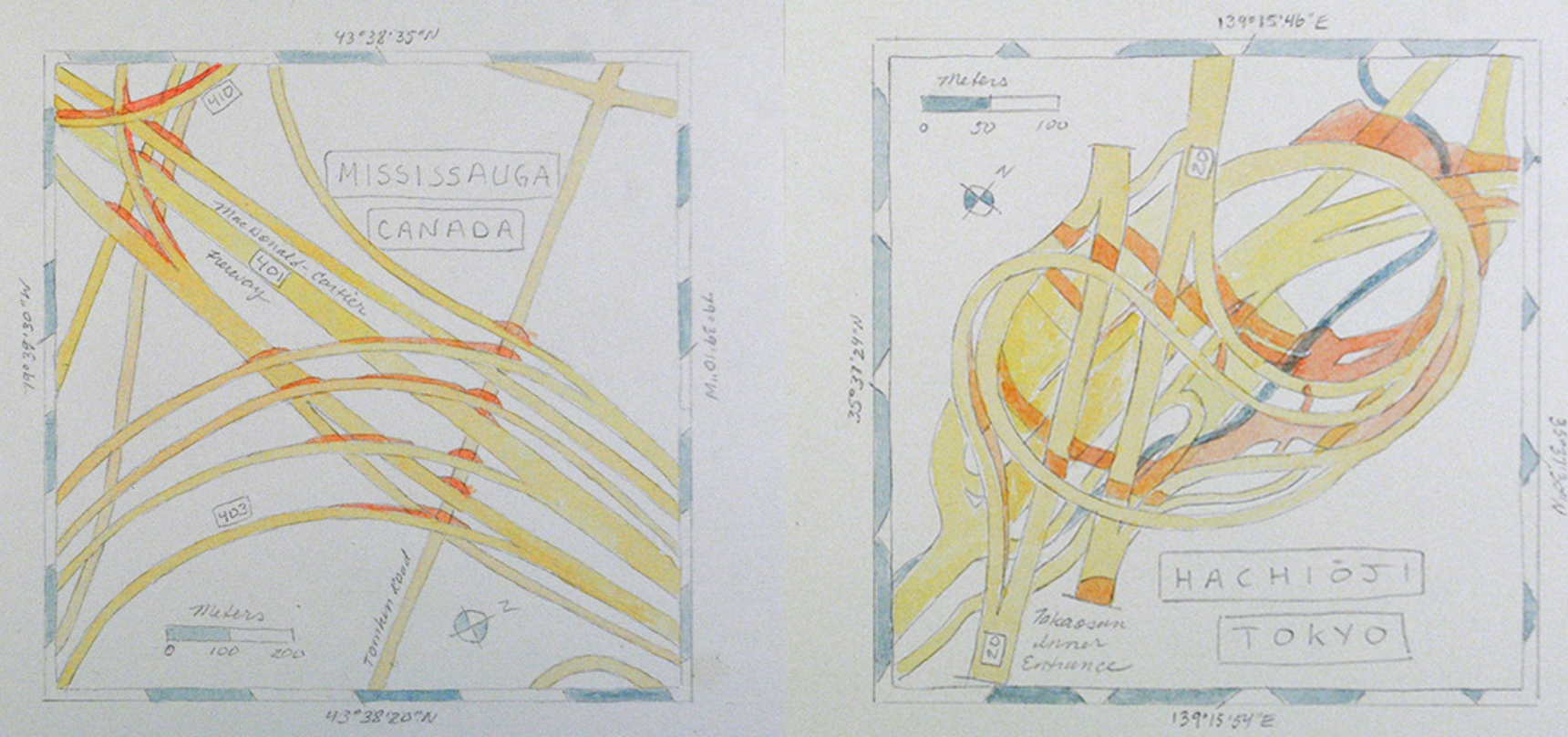

Crosshatching roads in Ontario and knotted roads in Japan.

These are all observations that I doubt I would have come by if I hadn't made these maps by hand. When you draw a map with software, you decide the symbology of a layer.
When you draw a map by hand, you consider every mark, if only for a moment, and you spend time with the shapes of things. Associations have time to evolve.
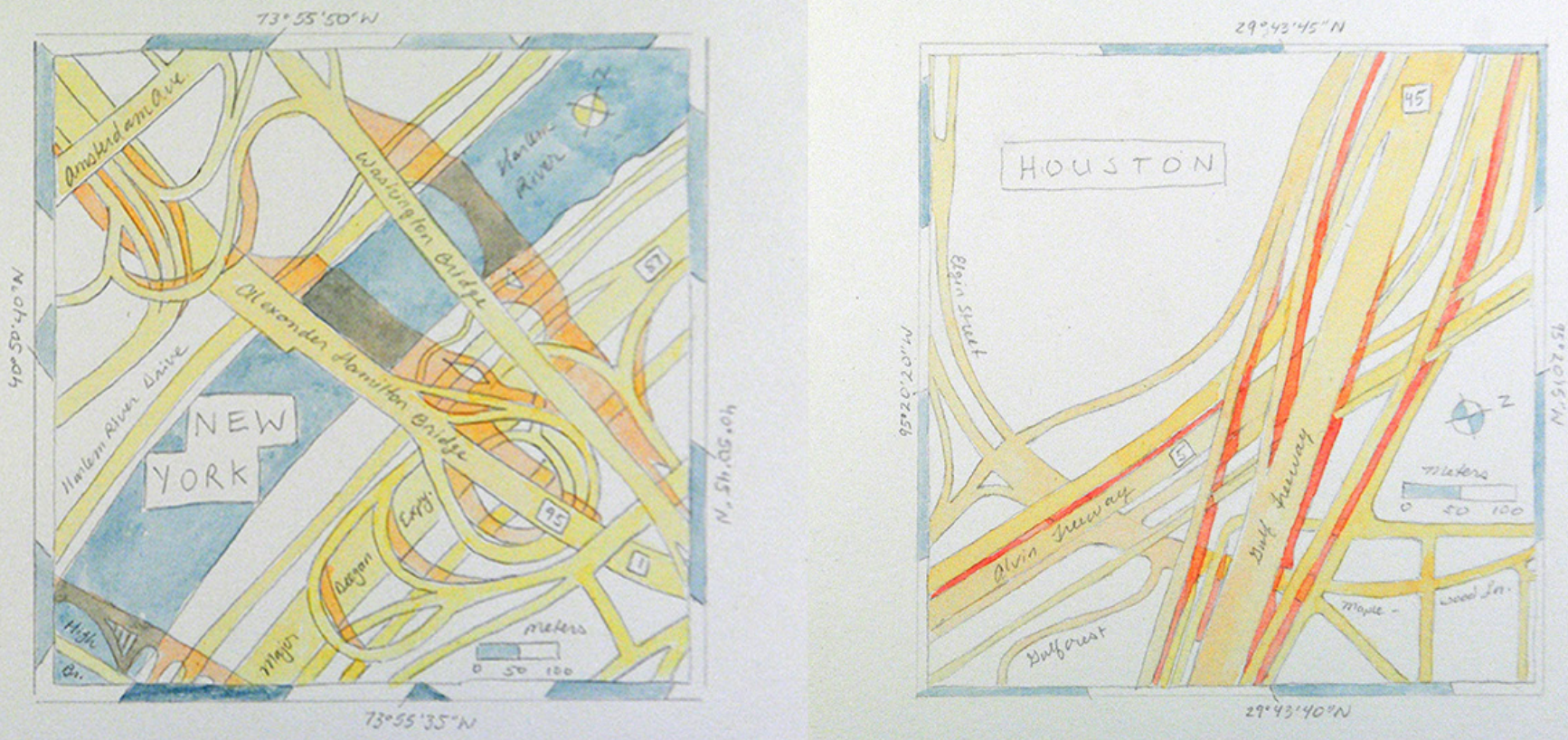

Highway interchanges in New York and Houston. 
Hand-drawn maps, and maps with hand-drawn elements, delight the viewer with their imperfections and individuality. But they also offer advantages to the cartographer:

- Simplicity and clarity of design is more strongly enforced, especially in media like woodcut or embroidery, where thin lines are not possible.

- A closer and more natural integration of map and illustration is possible.

When I decided to pursue cartography as a career, most of my artist friends commiserated that "you probably don't get to make maps by hand anymore."

I thought that was very silly. Maps had to be detailed and accurate. I could easily imagine spending hundreds of hours painstakingly drawing roads and rivers and labels, only to jump at the sound of the doorbell and smear ink all over the map. In a painting, I might be able to creatively work that streak into the final piece. Not so the map. I hadn't made any maps yet, but I was pretty convinced that I'd prefer to make them with computers.

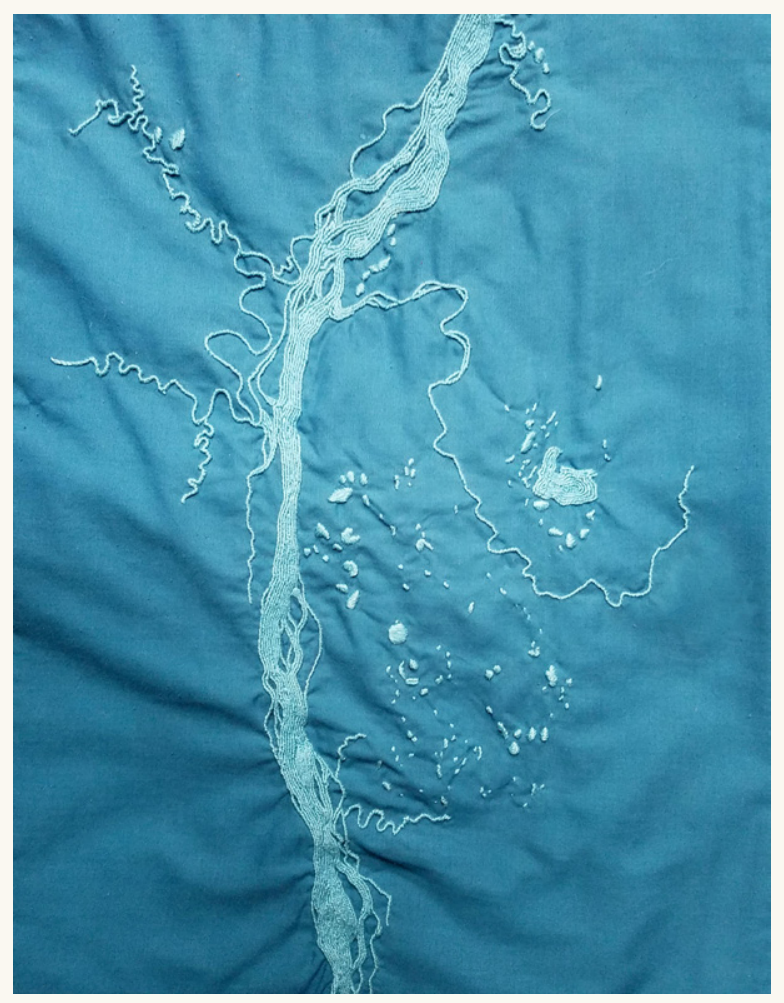

Embroidered map of the Lena River, $12 \times 18$

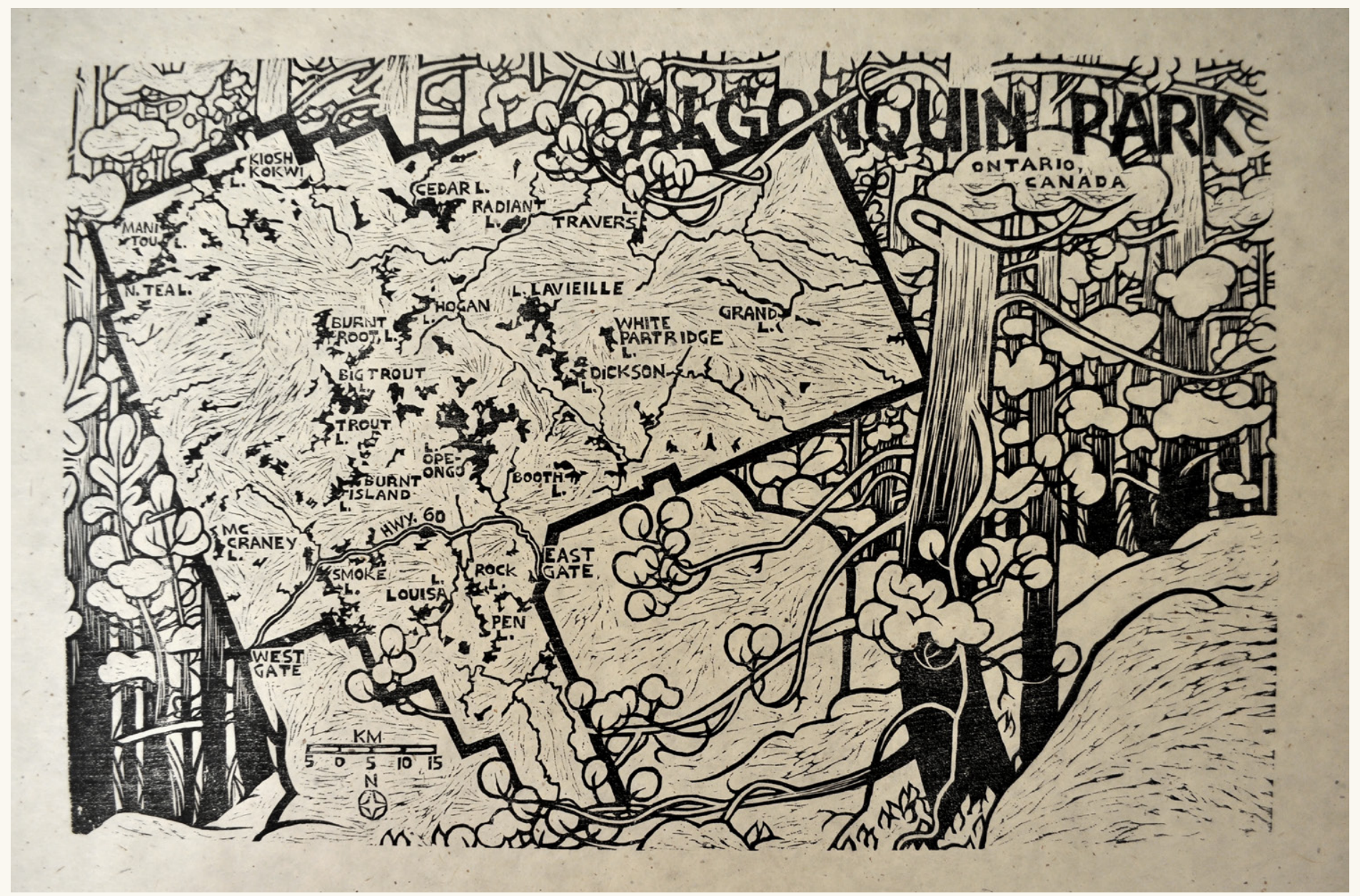






Detail of a map of mines in the Mojave Desert. Hachures were drawn by hand, and all other elements were created in ArcGIS Pro.

Fast forward seven years and I'm still very happy that I don't have to make maps by hand. This is work, after all, and efficiency matters. But making maps by hand has enhanced this work, and in particular, I've found that mixing the two techniques gives me the best of both worlds: with a pen I have the flexibility and variability to draw mountains and textures that bring the map alive, and with software I have Ctrl-Z, blend modes, and projections.

My current approach to this work goes something like this:

1. I plan the map using GIS software such as ArcGIS Pro. I choose the layers, scale, extent, projection, and rotation of the map. I also plan out symbology, but execute it only in broad strokes, in black and white, since multiple colors can make the next step difficult.



2. I tape some paper to my computer screen and trace the map. Some symbols I trace directly from Hand-drawn map before any post-processing. the draft map, while others I draw in with new 




Details of a map drawn for National Geographic, after processing in Photoshop, before labels and other text were added by Christina Shintani.

patterns. In some cases (pictorial mountains), I merely mark a location to draw on later.

3. I scan the map, and use Photoshop and Illustrator to clean up mistakes, re-colour lines, and add labels.

Last winter I worked on a commission for National Geographic of the ten longest rivers in the world, which gave me an opportunity to hone this technique. I used QGIS to plan the layout. Getting projections and scales correct and making sure all ten rivers could fit on the same page would have been a nightmare to plan on paper. But the individually-formed mountain drawings, tapered rivers, and stippled cities would have been frustrating to draw with software. Finishing the drawing in Photoshop and Illustrator allowed me to make the edits required without (always) having to redraw by hand.
I have mentioned a few of the aesthetic benefits of mapping by hand. However, I believe that the greater benefit is the experience and knowledge gained by the cartographer during the long, slow process. When you draw with software, the entire layer appears at once, and you merely decide the symbol properties. When you draw by hand, you decide each mark, and this is where I experience the true delight of geography. Without drawing every mark I never would have reason to consider why a road is depicted in one reference but not another, how some rock barrens streak across a peninsula in a particular pattern, how settlement patterns on one side of a border differ from those on another, or even just the loveliness of the shape of an unnamed lake.

When I draw geographies by hand, I'm forced to slow down and become acquainted with them. Driving through your town may be convenient and even necessary. But you'll only get to know the place if you walk. 